\title{
Performance Evaluation of Adaptive Virtual Machine Load Balancing Algorithm
}

\author{
Prof.Meenakshi Sharma \\ CSE Department SSCET Badhani \\ Pathankot, India
}

\author{
Pankaj Sharma \\ CSE Department SSCET Badhani \\ Pathankot, India
}

\begin{abstract}
The conception of Cloud computing has not only reshaped the field of distributed systems but also extend businesses potential. Load balancing is a core and challenging issue in Cloud Computing. How to use Cloud computing resources efficiently and gain the maximum profits with efficient load balancing algorithm is one of the Cloud computing service providers' ultimate goals. In this paper firstly an analysis of different Virtual machine(VM) load balancing algorithms was done, a new VM load balancing algorithm has been proposed and implemented in Virtual Machine environment of cloud computing in order to achieve better response time and cost.
\end{abstract}

Keywords-Virtual machine; load balancing; cloudsim.

\section{INTRODUCTION}

Cloud computing is a fast growing area in computing research and industry today. It has the potential to make the new idea of 'computing as a utility' in the near future. The Internet is often represented as a cloud and the term "cloud computing" arises from that analogy. Cloud computing is the dynamic provisioning of IT capabilities (hardware, software, or services) from third parties over a network [7]. It is generally supposed that there are three basic types of cloud computing: Infrastructure as a Service (IaaS), Platform as a Service (PaaS) and Software as a Service (SaaS) [1].

In IaaS grids or clusters, virtualized servers, memory, networks, storage and systems software are delivered as a service. Perhaps the best known example is Amazon's Elastic Compute Cloud (EC2) and Simple Storage Service (S3), IaaS Provide access to computational resources, i.e. CPUs. And also Provide (managed and scalable) resources as services to the user [7]. PaaS typically makes use of dedicated APIs to control the behavior of a server hosting engine which executes and replicates the execution according to user requests .E.g Force.com, Google App Engine. Software as a Service (SaaS) Standard application software functionality is offered within a cloud. Examples: Google Docs, SAP Business by design.Load balancing is one of prerequisites to utilize the full resources of parallel and distributed systems. Load balancing mechanisms can be broadly categorized as centralized or decentralized, dynamic or static, and periodic or non-periodic. Physical resources can be split into a number of logical slices called Virtual Machines (VMs).

All VM load balancing methods are designed to determine which Virtual Machine assigned to the next cloudlet [11]. This document introduce a new VM load balancing algorithm and compare the performance of this algorithms with the already existing algorithms like throttled and active monitoring VM load balancer [11]. Section III introduce the problem formulation, section IV include the purpose algorithm of the problem and result in section $\mathrm{V}$

\section{EXISTING VM LOAD BALANCER}

Virtual machine enables the abstraction of an Operating System and Application running on it from the hardware. The interior hardware infrastructure services interrelated to the Clouds is modelled in the simulator by a Datacenter element for handling service requests. These requests are application elements sandboxed within VMs, which need to be allocated a share of processing power on Datacenter's host components. DataCenter object manages the data center management activities such as VM creation and destruction and does the routing of user requests received from User Bases via the Internet to the VMs. The Data Center Controller [11] uses a VmLoadBalancer to determine which VM should be assigned to the next request for processing. Most common Vmloadbalancer are throttled and active monitoring load balancing algorithms.

\section{A. Throttled load balancer}

It maintain a record of the state of each virtual machine (busy/ideal), if a request arrive concerning the allocation of virtual machine, throttled load balancer send the ID of ideal virtual machine to the data center controller and data center controller allocates the ideal virtual machine.

\section{B. Active monitoring load balancer}

Active VM Load Balancer maintains information about each VMs and the number of requests currently allocated to which VM. When a request to allocate a new VM arrives, it identifies the least loaded VM. If there are more than one, the first identified is selected. ActiveVmLoadBalancer returns the VM id to the Data Center Controller; the data Center Controller sends the request to the VM identified by that id.DataCenterController notifies the ActiveVmLoadBalancer of the new allocation.

\section{PROBLEM FORMULATION}

In this paper a study of various virtual machine load balancing algorithms in cloud computing environment is done. The algorithms are round robin, throttled load balancer and active monitoring load balancer. A new algorithm has been 
proposed after modifying the throttled load balancing algorithm in Virtual Machine environment of cloud computing in order to achieve better response time, processing time and cost.

\section{Proposed Vm LoAD BALANCING AlgORITHM}

The Proposed VM Load balancing algorithm is divided into three phases.

The first phase is the initialization phase, where in the expected response time of each VM has been found.

Second Phase finds the efficient VM (VM having less response time), Last Phase returns the ID of efficient VM to datacenter controller.

- Efficient algorithms find expected response time of each Virtual machine.

// expected response time find with the help of resource info program

- When a request to allocate a new VM from the Datacenter Controller arrives, Algorithms find the most efficient VM (efficient VM having least loaded, minimum expected response time) for allocation.

- Proposed algorithms return the id of the efficient VM to the Datacenter Controller.

- Datacenter Controller notifies the new allocation

- Proposed algorithm updates the allocation table increasing the allocations count for That VM.

- When the VM finishes processing the request and the DataCenerController receives the Response. Datacenter controller notifies the efficient algorithm for the VM de-allocation.

- $\quad$ Start from step 2

The proposed algorithm finds the expected Response Time of each Virtual Machine because each virtual machine is of heterogeneous platform, the expected response time of each virtual machine can be found with the help of the following formula:

$$
\text { Response Time }=\text { Fin }_{\mathrm{t}}-\mathrm{Arr}_{\mathrm{t}}+\text { TDelay }
$$

Where $\operatorname{Arr}_{t}$ is the arrival time of user request and $\mathrm{Fin}_{t}$ is the finish time of user request and the transmission delay can be determined using the following formula:

$$
\text { TDelay }=\text { Tlatency }+ \text { Ttransfer }
$$

Where TDelay is the transmission delay, $\mathrm{T}$ latency is the network latency and $\mathrm{T}$ transfer is the time taken to transfer the size of single request from source location to destination.

Ttransfer $=\mathrm{D} /$ Bwperuser

Bwperuser $=$ Bwtotal $/ \mathrm{Nr}$

Where Bwtotal is the total available bandwidth and $\mathrm{N}_{\mathrm{r}}$ is the number of user requests currently in transmission. The Internet Characteristics also keeps track of the number of user requests in-flight between two regions for the value of $\mathrm{N}_{\mathrm{r}}$.

\section{EXPERIMENTAL RESULT}

Proposed algorithm is implemented with the help of simulation packages like CloudSim and cloudSim based tool [11]. Java language is used for implementing VM load balancing algorithm.

We Assume that the application has been deployed in one data center having 50 virtual machines (with $1024 \mathrm{Mb}$ of memory in each VM running on physical processors capable of speeds of 100 MIPS) where the parameter values are as under:

TABLE I. PARAMETER VALUE

\begin{tabular}{|l|l|}
\hline Parameter & value \\
\hline Data Center OS & Window 7 \\
\hline VM Memory & $1024 \mathrm{mb}$ \\
\hline Data Center Architecture & X86 \\
\hline Service Broker Policy & Optimize Response Time \\
\hline VM Bandwidth & 1000 \\
\hline
\end{tabular}

Followings are the experimental results based on Efficient VM Load Balancing Algorithm:

\begin{tabular}{|c|c|c|c|}
\hline \multicolumn{4}{|c|}{$\begin{array}{l}\text { Overall Avg Response Time With Efficient VM Load Balancin } \\
\text { Algorithms }\end{array}$} \\
\hline \multirow{2}{*}{$\begin{array}{l}\text { Overall } \\
\text { Response } \\
\text { Time }\end{array}$} & $\operatorname{Avg}(m s)$ & $\operatorname{Min}(m s)$ & $\operatorname{Max}(m s)$ \\
\hline & 171.43 & 35.06 & 618.14 \\
\hline \multicolumn{4}{|c|}{ Cost with Efficient Load Balancing Algorithm } \\
\hline \multirow[t]{2}{*}{ Cost } & $V M \operatorname{Cost} \$$ & $\begin{array}{l}\text { Data Transfer } \\
\text { Cost } \$\end{array}$ & Total Cost\$ \\
\hline & 240.11 & 1.94 & 242.05 \\
\hline
\end{tabular}

TABLE II. RESULT DETAIL

TABLE III. COMPARISON OF AVG RESPONSE TIME OF VM LOAD BALANCING ALGORITHMS.

\begin{tabular}{|l|l|l|l|}
\hline $\begin{array}{l}\text { Response } \\
\text { Time(ms) }\end{array}$ & $\begin{array}{l}\text { Throttled } \\
(\mathbf{m s})\end{array}$ & $\begin{array}{l}\text { Active } \\
\text { Monitoring } \\
\text { (ms) }\end{array}$ & $\begin{array}{l}\text { Efficient } \\
\text { (ms) }\end{array}$ \\
\cline { 2 - 4 } & 263.14 & 264.02 & 171.43 \\
\hline
\end{tabular}

Fig.1 shows the graphical representation of average Response time of VM load balancing algorithms. In our experiments, Average response Time of three VM load balancing algorithms was not same.

This experiment notifies that if we select an efficient virtual machine then it affects the overall performance of the cloud Environment. Fig 1 represent the average response time of each VM load balancing algorithm. 


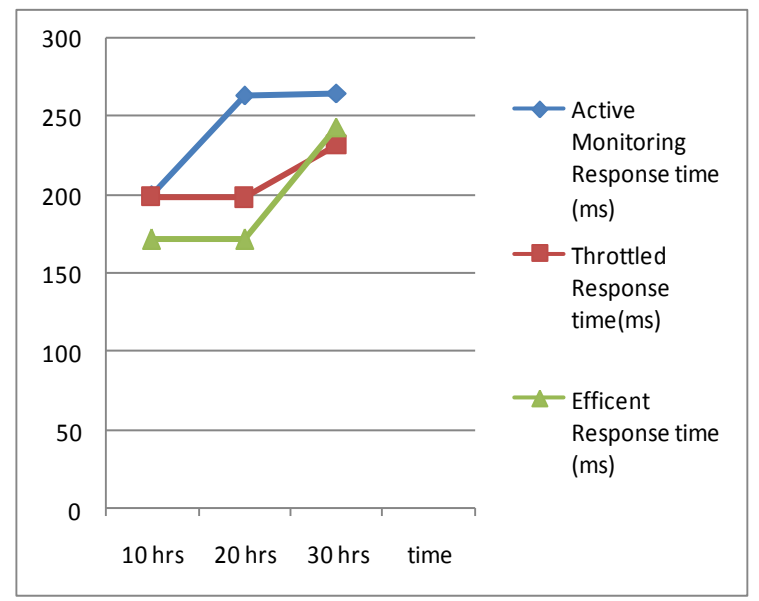

Figure 1. Comparison of Avg Response Time of VM Load balancing Algorithms.

\section{CONCLUSION}

In this paper, a new VM load balancing algorithm is proposed which is implemented in CloudSim, an abstract cloud computing environment using java language. Proposed algorithm finds the expected response time of each resource (VM) and sends the ID of virtual machine having minimum response time to the data center controller for allocation to the new request. According to this experiment, we conclude that if we select a efficient virtual machine then it effects the overall performance of the cloud Environment and also decreases the average response time.

\section{REFERENCES}

[1] CLOUD COMPUTING MADE EASY by Cary Landis and Dan Blacharski,version 0.3

[2] Ioannis Psoroulas, Ioannis Anagnostopoulos, Vassili Loumos and Eleftherios Kayafas. "A Study of the Parameters Concerning Load Balancing Algorithms" IJCSNS International Journal of Computer Science and Network Security, VOL.7 No.4, April 2007,Page No 202214 .

[3] Sandeep Sharma, Sarabjit Singh, and Meenakshi Sharma "Performance Analysis of Load Balancing Algorithms" World Academy of Science, Engineering and Technology 38,2008 page no 269- 272.

[4] Luqun Li ," An Optimistic Differentiated Service Job Scheduling System for Cloud Computing Service Users and Providers" Third International Conference on Multimedia and Ubiquitous Engineering,IEEE Explore , 4-6 June 2009, Page No 295-299.

[5] Rodrigo N. Calheiros, Rajiv Ranjan, César A. F. De Rose, and Rajkumar Buyya, "Modeling and Simulation of Scalable Cloud Computing Environment and CloudSim Toolkit: Challenges and opportunities".IEEE Explore International Conference on high performance Computing And simulation, 21-24 June 2009 Page No 111.
[6] QI CAO , ZHI-BO WEI ,WEN-MAO GONG.” An Optimized Algorithm for Task Scheduling Based On Activity Based Costing in Cloud Computing". International Conference on Bioinformatics and Biomedical Engineering,IEEE Explore 14 July 2009 Page No 1-3.

[7] Bhasker Prasad Rimal, Eummi Choi, Lan Lump "A Taxonomy and Survey of Cloud Computing System" 5th International Joint Conference on INC,IMS and IDC,IEEE Explore 25-27 Aug 2009,Page No 44-51.

[8] Yi Zhao, Wenlong Huang,“ Adaptive Distributed Load Balancing Algorithm based on Live Migration of Virtual Machines in Cloud" IEEE 5th International Joint Conference on INC,IMS and IDC, 25-27 Aug 2009,Page No 170-176

[9] Huai Zhang, Shufen Zhang, Xuebin Chen, Xiuzhen Huo," Cloud Computing Research and Development Trend" Second International Conference on Future Networks, 22-24 Jan 2010, Page No 93-97.

[10] Martin Randles, David Lamb, A. Taleb-Bendiab, A Comparative Study into Distributed Load Balancing Algorithms for Cloud Computing, IEEE 24th International Conference on Advanced Information Networking and Applications Workshops 20-23 April 2010, Page No 551-556.

[11] Bhathiya Wickremasinghe, Rodrigo N. Calheiros, and Rajkumar Buyya. "CloudAnalyst: A CloudSim-based Visual Modeller for Analysing Cloud Computing Environments and Applications",20-23 April 2010 Page No 446-452.

[12] Zehua Zhang and Xuejie Zhang," A Load Balancing Mechanism Based on Ant Colony and Complex Network Theory in Open Cloud Computing Federation" 2010 2nd International Conference on Industrial Mechatronics and Automation. 30-31 May 2010 Page No 240-243.

[13] Thomas A. Hen zinger Anmol V. Singh Vasu Singh Thomas Wies Damien Zufferey, FlexPRICE:Flexible Provisioning of Resources in a Cloud Environment, IEEE 3rd International Conference on Cloud Computing 5-10 july 2010.Page No 83-90.

[14] Shu-Ching Wang, Kuo-Qin Yan *(Corresponding author), Wen-Pin Liao and Shun-Sheng Wang "Towards a Load Balancing in a Three-level Cloud Computing Network"IEEE 3rd International Conference on Computer Science \& Information Technology 9-11 July 2010, Page No 108-113.

[15] Hai Zhong1, 2, Kun Tao1, Xuejie Zhang," An Approach to Optimized Resource Scheduling Algorithm for Open-source Cloud Systems" The Fifth Annual ChinaGrid Conference 16-18 July 2010, Page No 124-129

[16] Yoshitomo MURATA, Ryusuke EGAWA, Manabu HIGASHIDA, Hiroaki KOBAYASHI," A History-Based Job Scheduling Mechanism for the Vector Computing Cloud" 2010 10th Annual International Symposium on Applications and the Internet.19-23 July 2010.Page No 125-128

[17] Andrew J. Younge, Gregor von Laszewski, Lizhe Wang, Sonia LopezAlarcon, Warren Carithers," Efficient Resource Management for Cloud Computing Environments" International Conference on Green Computing.15-18 Aug 2010, Page No 357-364

[18] LIU Jia, HUANG Ting-Lei," Dynamic Route Scheduling for Optimization of Cloud Database. International Conference on Intelligent Computing and Integrated System, 22-24 Oct 2010,Page No 680-682

[19] Jinhua Hu, Jianhua Gu, Guofei Sun,Tianhai Zhao," A Scheduling Strategy on Load Balancing of Virtual Machine Resources In Computing Environment" 3rd International Symposium on Parallel Architecture, Algorithms and Programming IEEE Explore 18-20 Dec 2010,page no. 89-96. 